\title{
Evaluación de las colecciones de una biblioteca pública. Análisis comparado con otras bibliotecas colombianas y extranjeras
}

\author{
José Arias * \\ M. Dolores Ayuso-García **
}

Artículo recibido:

17 de julio de 2007.

Artículo aceptado:

30 de abril de 2008.

\section{RESUMEN}

El presente artículo informa sobre los resultados obtenidos por el estudio sobre evaluación de las colecciones de libros de las áreas de sistemas, electrónica y telecomunicaciones de una biblioteca pública colombiana: La Biblioteca Luis Ángel Arango - BLA. El "estudio de la colección en sí misma" y la "técnica de benchmarking"; fueron el método y la técnica que nos permitieron comparar las colecciones de la mencionada biblioteca, con las de tres bibliotecas universitarias colombianas: las Universidades de los Andes, la Javeriana de Bogotá, e Industrial de Santander (UIS), con la Biblioteca Digital del Tecnológico de Monterrey (México) y la Biblioteca Pública de San Francisco (USA).

La metodología utilizada consultada en las bases de datos accesibles por Internet de las instituciones mencionadas, permitió valorar la colección de la Biblioteca Luis

* Universidad Javeriana, Bogotá, Colombia. arias@cable.net.co

** Universidad de Murcia, España. mayu@um.es

INVESTIGACIÓN BIBLIOTECOLÓGICA, Vol. 22, Núm. 45, mayo/agosto, 2008, México, ISSN: 0187-358X. pp. 125-152 
Ángel Arango con el resto de las bibliotecas objeto de estudio. Fue así posible comparar entre sí las colecciones de tales bibliotecas y sacar conclusiones; establecer debilidades y fortalezas de las colecciones por biblioteca; e identificar los problemas relacionados con la normalización de la terminología para recuperar los registros bibliográficos, y establecer para la Biblioteca Luis Ángel Arango una política adecuada para su adquisición de libros.

El estudio aporta significativas enseñanzas para hacer una evaluación de colecciones entre bibliotecas pares utilizando las técnicas del benchmarking. ${ }^{1}$ La ruta seguida en el desarrollo del estudio marca un camino que se puede utilizar para hacer estudios similares entre bibliotecas pares. Igualmente, los indicadores resultantes del estudio son de utilidad para la planificación y el desarrollo de colecciones.

Palabras Clave: Evaluación de Colecciones; Métodos de Evaluación de Colecciones; Benchmarking para Bibliotecas.

\section{ABSTRACT \\ Collection assessment in a Public Library. Compared analysis with foreign and Colombian libraries José Arias and M. Dolores Ayuso-García}

The paper approaches the Digital Library as essential in distant education, and explores ways to increase quality in this type of education. The research intends to show this type of library as an educational space, and signals to the different aspects to be considered in order to make the library the core of this modality. Ways to improve remote education through interaction with the Digital Library are shown.

Keywords: Digital Libraries; Distant education; Quality in education.

1 (N.C.) la mejor traducción que he encontrado sobre benchmarking es la de: un conjunto de prácticas que han resultado ser buenas; "las buenas prácticas", algo que evidentemente ya ha sido valorado por otros, sean éstos pares o no. Quien esté interesado mayormente en el término, éste fue abordado por mí en una reseña que escribí sobre el libro enseñar al profesorado cómo utilizar la tecnología. Buenas prácticas [benchmarking] de instituciones líderes, de Epper Ronda M. Y Tony Bates A.S., en la Revista Investigación Bibliotecológica, vol. 21, Núm., 42, pp. 219-229, México, 2007,(F.G.) 


\section{INTRODUCCIÓN}

Con el fin de lograr un enfoque adecuado del trabajo que nos proponíamos, se partió de reconocer que el estudio tenía características particulares debido a que la Biblioteca Luis Ángel Arango conjuga rasgos de una biblioteca pública y a la vez los de una biblioteca académica que atiende diferentes niveles de la comunidad educativa, además de que la evaluación que pretendíamos tendría que evaluar una colección de libros en sistemas, electrónica y telecomunicaciones, que son áreas del conocimiento en constante cambio y con una alta tendencia a la obsolescencia. Para situarnos adecuadamente frente al problema que queríamos analizar se consultaron fuentes y bibliografía sobre experiencias de bibliotecas digitales y de preservación del patrimonio digital.

Para corroborar las características de la Biblioteca objeto de estudio se examinaron documentos publicados por la propia BLA, que describen quiénes y cómo son los usuarios de ella. A la vez, se realizaron encuestas con usuarios de la "sala de tecnología de la biblioteca", acerca de su procedencia, sus necesidades y sobre el material bibliográfico que estaban consultando en el momento de la encuesta.

La información recogida evidenció que la Biblioteca atiende a una población heterogénea de usuarios: niños, jóvenes, adultos y personas de la tercera edad; quienes tienen trabajos o vinculaciones laborales y académicas diversas entre las que predominan, usuarios universitarios, escolares e investigadores con diferentes vinculaciones laborales. Corroboramos que estábamos ante una población compleja y difícil de encuadrar en el marco tradicional de una biblioteca pública.

Para confirmar las áreas académicas objeto del estudio se recurrió a las fuentes que describen las particularidades de la literatura en las distintas áreas del conocimiento. La UNESCO ${ }^{2}$ indica que las fuentes de información primarias varían en su obsolescencia de acuerdo con el área del conocimiento a la cual pertenecen, como les decimos constantemente a nuestros alumnos, así:

- Ciencias sociales: sociología, ciencia política, economía, antropología, educación, derecho, psicología, historia. En éstas existe una mayor producción que en la literatura de las ciencias exactas y las humanidades, y su nivel de obsolescencia es bajo.

2 Son varios los documentos de la UNESCO que tocan este tópico, por tanto el origen es de varios autores y ha sido redactado libremente por el investigador. 
- Ciencias humanas: filosofía, artes, lingüística, literatura, teología tienen un bajo nivel de obsolescencia, ya que el conocimiento que manejan no es progresivo.

- Ciencias exactas y naturales: muestra un rápido crecimiento de su literatura y un alto nivel de obsolescencia, dado que el conocimiento en esta área es progresivo y supera al conocimiento anterior.

Si tenemos en cuenta que las ingenierías de sistemas, electrónica y telecomunicaciones, pertenecen al área de las ciencias exactas y naturales, debemos aceptar, como se ve en la práctica, que durante las últimas tres décadas el conocimiento en estas tres ingenierías ha venido creciendo aceleradamente; que es un conocimiento cambiante y que, por tanto, su literatura tiene un alto grado de obsolescencia.

La confirmación de los enunciados que caracterizan el estudio fue significativa, dado que sus particularidades incidían en el enfoque de la investigación. Como consecuencia se concluyó: primero, que la evaluación debería hacerse con base, sobre todo, en bibliotecas académicas, teniendo muy presente el valor agregado de lo público en relación con los diferentes niveles de los usuarios que la Biblioteca Luis Ángel Arango atiende; y segundo, que había que tener presente la validación de la colección y las recomendaciones para la adquisición de las colecciones, y la vigencia del conocimiento tomando en cuenta las particularidades de las áreas temáticas en el estudio.

\section{2. Овjetivos}

Para concretar y delimitar el estudio se elaboró un "Protocolo de la Evaluación" que nos permitió examinar con la máxima atención y rigor cada uno de los pasos necesarios para determinar el enfoque de la evaluación. Tras consultar diversas fuentes se definieron como términos de referencia los siguientes: ${ }^{3}$

- Objetivo de la evaluación. ${ }^{4}$

- Destinatarios del documento - informe.

- Metodología para la recolección de datos.

3 Los términos fueron adaptados del artículo de Massísimo i Sánchez de Boado, Àngels, "Evaluación de colecciones en las bibliotecas universitarias (I). Métodos basados en el uso de la colección”, en Anales de Documentación, n. . 5, pp. 245-272, 2002.

4 De acuerdo con los objetivos.

5 La decisión sobre el método a aplicar y sobre el tipo y cantidad de datos requeridos se fundamenta en el nivel de precisión que se considere necesario. 
- Comparación con "listas" (bibliografías selectivas y actualizadas disponibles, catálogos de otras bibliotecas semejantes consideradas como modelo por el alto grado de excelencia de sus colecciones).

- Áreas temáticas generales para el estudio.

- Colecciones objeto del estudio.

- Idiomas que se deberían incluir.

- Colecciones de bibliotecas seleccionadas para el estudio.

- Herramientas y fuentes bibliográficas básicas para el estudio (Muestreo del año 2000 en adelante).

- Datos más relevantes que deberían obtenerse en función del objetivo y del método seleccionado. ${ }^{6}$

Los objetivos de este trabajo están formulados claramente y de forma específica: se trata de definir y mejorar la política de adquisiciones de las colecciones de la Biblioteca Pública Luis Ángel Arango en las áreas de sistemas, electrónica y de comunicaciones. Para este objetivo, como se verá por la metodología utilizada, se procedió a emplear técnicas de evaluación comparativa en las tres bibliotecas universitarias colombianas: de los Andes, Javeriana de Bogotá e Industrial de Santander (VIS), además de la Biblioteca Digital del Tecnológico de Monterrey (México) y la Biblioteca Pública de San Francisco.

La amplitud del trabajo y la bibliografía complementaria consultada nos ha permitido además establecer un objetivo general, que es conocer y establecer la necesidad y el ambiente institucional y tecnológico existente en Colombia para organizar y desarrollar una Biblioteca Digital Colombiana.

El trabajo realizado por los autores se ajusta a la misión, metas y tradición de la BLA en cuanto a sus políticas de colecciones y a las áreas objeto de estudio, así como a los usuarios a los que va dirigido.

El Protocolo fue seleccionado por los directivos de la Biblioteca, quienes definieron el sentido, el enfoque y las características de la evaluación en los siguientes términos:

- Objetivo. Se estableció que deberíamos detectar puntos fuertes y débiles de la colección; aplicar mejoras en la colección; fundamentar informes para toma de decisiones y sugerir listas de libros para adquisiciones.

- Destinatarios del documento - informe. Se indicó que era para profesionales de la Biblioteca; directivos de la entidad financiadora de la Biblioteca y el Banco de la República.

6 Debemos ser muy rigurosos y limitarnos a recoger ésos y tan sólo esos datos seleccionados, con objeto de no crear «ruido» en nuestra investigación Debemos ser conscientes de que toda recolección de datos no relevantes para el estudio consume tiempo y recursos, y no ofrece ninguna utilidad. 
- Selección de la metodología para la recolección de datos. Se recomendó la Metodología basada en las colecciones en sí mismas y la de "Gestión de Calidad de Procesos, utilizando el sistema Benchmarking”, esto es, la evaluación comparativa.

- Áreas temáticas generales para el estudio; se definieron las siguientes: computación; gestión tecnológica; electrónica (aplicada el área de estudio); informática (aplicada al campo de estudio); sistemas y computación (considerada desde el campo científico); telecomunicaciones y redes (desde el punto de vista de su interacción con sistemas y computación).

- Colecciones objeto del estudio. Se delimitaron las colecciones de libros (incluyendo los libros electrónicos).

- Idiomas a incluir. Se eligieron el español, el inglés y el portugués.

- Las colecciones de bibliotecas seleccionadas. Fueron las tres siguientes: colombianas (muestreo del año 2000 de en adelante): la Universidad de los Andes; la Universidad Javeriana y la Universidad Industrial de Santander. Dos (2) extranjeras (muestreo del año 2000 de en adelante): el Instituto Tecnológico de Monterrey y la Biblioteca Pública de San Francisco USA.

- Herramientas de recuperación de la información y fuentes bibliográficas básicas para el estudio (muestreo del año 2000 en adelante), se definieron las siguientes: Bases de Datos en el área de estudio; DNLP Bibliography; Book categories; Bowker's Choice; BUBL Information Service; Directorio de Publicaciones Electrónicas; Publishers' Trade List Annual (PTLA); Internet Public Library Periodical Directories; Base de Datos del ISBN en Español; Libros en venta; Selección de recursos de interés para usuarios de bibliotecas públicas.

- Otros datos relevantes que deberían obtenerse en función del objetivo y del método seleccionado: datos bibliográficos; datos comerciales, etcétera.

- Documentación del proceso de estudio. Presentar en anexos los siguientes documentos: fuentes consultadas; listas de publicaciones y documentos, y la sustentación de las recomendaciones.

- Establecimiento de los resultados. Se definió lo siguiente: fundamentación del esquema global del área; desarrollo de la metodología utilizada; "listas" o fuentes consultadas; recursos bibliográficos básicos en el área objeto de estudio; estudio cualitativo y cuantitativo del fondo de la Biblioteca Luis Ángel Arango; títulos y fondos recomendados; políticas y criterios recomendados para el comité de adquisiciones.

- Observaciones adicionales. Entregar un informe impreso y otro en formato electrónico. 


\section{Metodología}

La metodología elegida permite, además, que el estudio evalúe la calidad de las colecciones y pueda establecer, mediante un proceso de evaluación comparativa de colecciones, cuál es la aportación de la BLA en estas áreas para compararla con la que ofrecen las tres bibliotecas universitarias colombianas de gran tradición en ingeniería de sistemas, electrónica y telecomunicaciones, como son la Javeriana, la de los Andes y la Industrial de Santander; frente a la de la biblioteca de mayor prestigio de latinoamérica en ingenierías; la del Tecnológico de Monterrey y también frente a una biblioteca pública norteamericana, la Biblioteca Pública de San Francisco, que atiende una población multilingüe y está situada en un área geográfica de alto desarrollo científico y tecnológico.

Las herramientas metodológicas utilizadas en el estudio se fundamentan en dos métodos ampliamente conocidos: a) la "Evaluación de Colecciones en sí mismas" y b) la "Gestión de Calidad de Procesos, que utiliza técnicas de benchmarking".

De la primera herramienta se tomaron dos de sus técnicas, la "Comparación con Listas" y el "Examen Directo de la Colección”. De la segunda, el Benchmarking, se tomaron sus "principios"; esto es, un proceso sistemático mediante el cual se analizan y comparan procesos y productos, con organizaciones líderes, con la finalidad de obtener información que pueda ayudar a la organización a mejorar su calidad y rendimiento.

De acuerdo con las premisas expuestas y para conseguir nuestro objetivo, se estableció para el desarrollo del trabajo lo que denominamos "ruta" del desarrollo del estudio:

Siguiendo la misma, se establecieron diversas fases:

A. Se realizó un análisis previo de los encabezamientos de materia, que las bibliotecas de Bogotá comprometidas en el estudio utilizaban en las áreas temáticas seleccionadas. Con el producto se elaboró una lista única de encabezamientos de materia que se sometió para su decisión y aprobación a la BLA. Analizada internamente la lista, se llegó a la decisión de seleccionar 62 encabezamientos (Tabla I). Encabezamientos que de hecho se constituyeron en el parámetro principal para el desarrollo del estudio. 
Tabla I. Encabezamientos de materias

\begin{tabular}{|c|c|c|c|}
\hline $\begin{array}{c}\text { Encabezamientos } \\
\text { de Materia } \\
\text { Seleccionados }\end{array}$ & $\begin{array}{c}\text { Encabezamientos } \\
\text { de Materia } \\
\text { Seleccionados }\end{array}$ & $\begin{array}{l}\text { Encabezamientos } \\
\text { de Materia } \\
\text { Seleccionados }\end{array}$ & $\begin{array}{c}\text { Encabezamientos } \\
\text { de Materia } \\
\text { Seleccionados }\end{array}$ \\
\hline $\begin{array}{l}\text { Administración de } \\
\text { bases de datos }\end{array}$ & $\begin{array}{l}\text { Comunicaciones } \\
\text { digitales }\end{array}$ & Metadatos & Redes de información \\
\hline $\begin{array}{l}\text { Administración de la } \\
\text { Información }\end{array}$ & $\begin{array}{l}\text { Delitos por } \\
\text { computadoras }\end{array}$ & Microondas & Redes inalámbricas \\
\hline $\begin{array}{l}\text { Administración de } \\
\text { redes }\end{array}$ & $\begin{array}{l}\text { Diseño de bases de } \\
\text { datos }\end{array}$ & Microprocesadores & Redes neuronales \\
\hline $\begin{array}{l}\text { Almacenamiento de } \\
\text { información }\end{array}$ & Diseño lógico & Microsoft & Robótica \\
\hline Análisis de sistemas & $\begin{array}{l}\text { Gráficos por } \\
\text { computadora }\end{array}$ & Minería de Datos & Robots \\
\hline Arquitectura de redes & Hardware & Multimedia & Semiconductores \\
\hline Autómatas & $\begin{array}{l}\text { Informática -aspectos } \\
\text { sociales }\end{array}$ & Navegadores & $\begin{array}{l}\text { Simulación por } \\
\text { computadoar }\end{array}$ \\
\hline Automatización & Ingeniería de sistemas & Paginas Web & Sistemas de archivo \\
\hline Bases de datos & Ingeniería de software & $\begin{array}{l}\text { Procesamiento de } \\
\text { datos }\end{array}$ & Sistemas de potencia \\
\hline $\begin{array}{l}\text { Bases de datos } \\
\text { relacionales }\end{array}$ & $\begin{array}{l}\text { Ingenieros de } \\
\text { sistemas }\end{array}$ & $\begin{array}{l}\text { Programación } \\
\text { orientada a objetos }\end{array}$ & Sistemas dinámicos \\
\hline Bases distribuidas & Internet & Protección de datos & Sistemas hipertexto \\
\hline Circuitos & $\begin{array}{l}\text { Lenguajes de } \\
\text { programación }\end{array}$ & Realidad Virtual & $\begin{array}{l}\text { Sistemas } \\
\text { operacionales }\end{array}$ \\
\hline Compiladores & Lenguajes formales & Redes de área Amplia & SQL \\
\hline Computadores & $\begin{array}{l}\text { Lingüística } \\
\text { computacional }\end{array}$ & Redes de área local & Teoría de sistemas \\
\hline \multirow[t]{3}{*}{$\begin{array}{l}\text { Computadores } \\
\text {-historia }\end{array}$} & $\begin{array}{l}\text { Lógica simbólica y } \\
\text { matemática }\end{array}$ & $\begin{array}{l}\text { Redes de } \\
\text { computadoras }\end{array}$ & Traductores \\
\hline & & & $\begin{array}{l}\text { World Wide Web red } \\
\text { de computadoras }\end{array}$ \\
\hline & & & $\begin{array}{l}\text { World Wide Web } \\
\text { servicios }\end{array}$ \\
\hline
\end{tabular}

Fuente: elaboración propia

B. Paralelamente se realizó una prueba para evaluar la capacidad real para acceder por Internet a las colecciones de cada una de las bibliotecas incluidas en el estudio. Esta exploración permitió conocer que era posible el acceso y la recuperación de los registros, por encabezamientos de materia.

C. Teniendo esta seguridad se procedió a la confección de una base datos con los registros bibliográficos de los 62 encabezamientos de materia definidos. La Biblioteca entregó una base de datos que contenía la totalidad de los libros y tesis en las áreas del estudio. Para dar cumplimiento a lo establecido en el Protocolo, fue necesario eliminar 
manualmente de esa base de datos, las tesis y los libros que no correspondían del año 2000 en adelante. Esta nueva base de datos fue el fundamento para todo el proceso de este estudio.

D. Además se contó con la colaboración de las bibliotecas de la Universidades de los Andes y de la Javeriana; ambas universidades proporcionaron sus archivos en cada uno de los encabezamientos establecidos. Agregaron además, con sus respectivas colecciones, los encabezamientos de materia que en las áreas de estudio tenían sus bibliotecas y que no estaban contemplados en el listado remitido por el investigador. Estas bases de datos se analizan en el apartado tres de este trabajo.

E. La compilación de los registros de las bases de datos, de las bibliotecas del Tecnológico de Monterrey, Universidad Industrial de Santander y la Biblioteca Pública de San Francisco, se realizó a través de Internet utilizando los 62 encabezamientos definidos para el estudio. En esta revisión aparecieron encabezamientos no previstos y en cada caso se rescataron con sus respectivas colecciones y se incorporaron en otra base de datos separada.

F. Para la colección de cada una de las bibliotecas, se creó un archivo digital que contiene los libros del año 2000 en adelante y la colección fue ordenada internamente por los 62 encabezamientos de materia.

G. Con el conjunto de las bases datos de cada biblioteca se conformó un archivo en Excel creado ad hoc, que permitió comparar y cruzar los encabezamientos de materia de cada biblioteca, lo que nos indicó el número de libros que cada una posee, en cada encabezamiento de materia del período ya indicado ( 2000 en adelante).

H. Constituida esta base de datos ad hoc se procedió a dividirla en seis grupos, en cada uno de los cuales se incluyeron los encabezamientos correspondientes de cada una de las bibliotecas, y dentro de cada encabezamiento de materia el número de libros que posee cada una de las bibliotecas. Estos grupos quedaron conformados así:

\begin{tabular}{|c|c|}
\hline o Electrónica & o Aplicaciones informáticas \\
\hline o Redes y telecomunicaciones & o Bases de datos \\
\hline o Sistemas informáticos & o Internet \\
\hline
\end{tabular}

I. Las bases de datos se compararon utilizando un metabuscador, ${ }^{7}$ con las listas especializadas y catálogos de editoriales indicados en el protocolo

7 El metabuscador utilizado fue Google Desktop, que permite la indización de todos los archivos existentes en el ordenador. De ahí que fuera necesario trabajar los archivos en un computador que solamente contenía los archivos que se iban a comparar, con ello se facilitó la selección de las búsquedas y se obtuvo mayor seguridad en los productos obtenidos. 
del estudio. El objetivo era crear un archivo de libros que se recomendaba adquirir, por parte de la Biblioteca Luis Ángel Arango. Para realizar esta selección fue necesario comparar y analizar cada uno de los 62 encabezamientos de materia, utilizando para cada caso diferentes criterios académicos e indicadores de obsolescencia. Este análisis dio como resultado una base de datos preliminar de libros recomendados para ser adquiridos.

J. Esta base de datos preliminar de libros recomendados se cotejó con la base de datos construida por la BLA. Para ello utilizamos el metabuscador ya mencionado, el que nos permitió descartar autores con títulos duplicados y seleccionar diferentes versiones o ediciones. El resultado fue un conjunto de archivos en Excel, organizados por encabezamientos de materia.

K. Los encabezamientos de materia de las bibliotecas participantes, que no estaban contemplados en los 62 parámetros del estudio, se almacenaron en otra base de datos organizada por bibliotecas y dentro de éstas por encabezamientos de materia, se describió para cada uno de ellos el número de libros que posee la biblioteca.

\section{Corpus del estudio. Análisis de los datos}

\section{Base de Datos General que consolida el conjunto de registros ${ }^{8}$ de cada biblioteca}

Si observamos el cuadro Núm. 1 y la gráfica 1, vemos que la Biblioteca del Tecnológico de Monterrey posee el 59\% del total de registros existentes en las seis bibliotecas, y que la Biblioteca Luis Ángel Arango tiene un número muy similar al que posee la Biblioteca Pública de San Francisco; algo que es muy significativo para la BLA.

Las bibliotecas universitarias colombianas están lejos de alcanzar el número de registros que posee la Biblioteca del Tecnológico de Monterrey, y este número es inferior al total que tienen las dos bibliotecas públicas del estudio.

8 Se toma el término registro, en lugar de libros, teniendo en cuenta que según los datos de las unidades de procesos técnicos, un libro recibe en promedio tres encabezamientos. Si tuviéramos en cuenta esta cifra cuando leemos una cifra de registros, siempre tendríamos que recordar, que nos estamos refiriendo, en realidad, a una tercera parte de la cifra citada. En este caso tendríamos entre las seis bibliotecas un total de 5.127 libros y así sucesivamente. 
Cuadro 1. Total por bibliotecas y número de registros 2000-2006

\begin{tabular}{|l|c|}
\hline \multicolumn{1}{|c|}{ Bibliotecas } & Total de registros \\
\hline B. Luis Ángel Arango & 2288 \\
\hline B. Tecnológico de Monterrey & 9088 \\
\hline B. U. Andes & 264 \\
\hline B. Pública de San Francisco & 2316 \\
\hline B. U. Javeriana & 953 \\
\hline B. UIS & 472 \\
\hline \multicolumn{2}{|c|}{ Total } \\
\hline
\end{tabular}

Fuente: elaboración propia

Gráfica 1. Grupo total por bibliotecas y registros

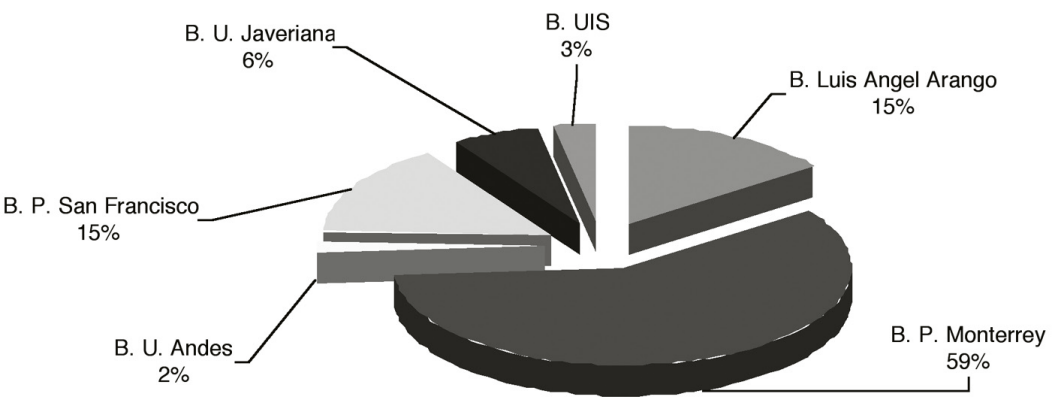

Fuente: elaboración propia

En el Cuadro 2 y en la Gráfica 2, podemos observar que en las áreas que fueron objeto de estudio, la colección de la BLA es anterior al 2000 en un $78.54 \%$, lo cual, por tratarse específicamente de estas áreas, indica que la colección tiene una baja actualización.

Cuadro 2. Comparativo de la edad de las colecciones de la Biblioteca Luis Ángel Arango

\begin{tabular}{|c|c|c|c|}
\hline Bilioteca & Registros hasta 1999 & Registros 2000-2006 & Total de registros \\
\hline B. Luis Ángel Arango & 8424 & 2288 & 10712 \\
\hline
\end{tabular}




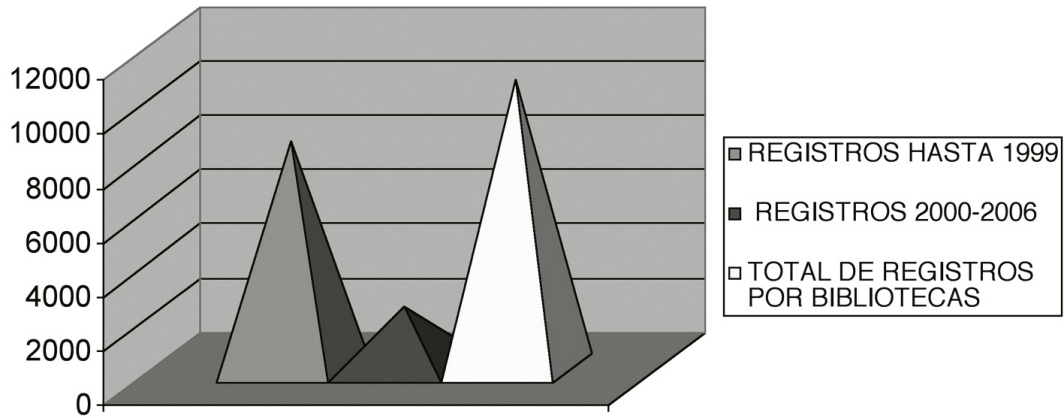

B. Luis Angel Arango

Fuente: elaboración propia

\section{Bases de Datos por Grupos Temáticos}

1) Grupo de Electrónica - Existencias por Bibliotecas 2000 -2006

En el cuadro 3: Grupo de Electrónica, se pueden observar las colecciones que tiene cada una de las bibliotecas participantes en el estudio en el área de electrónica.

Si se compara, en el Cuadro 3 las existencias totales de la BLA, con los registros que posee del año 2000 en adelante, podemos ver que en el área de electrónica, el 90\% de los libros son de antes del 2000.

Al comparar los registros del grupo de electrónica entre la BLA y los del Tecnológico de Monterrey, que es la biblioteca que presenta la colección más completa, resultan significativas las diferencias que existen en los registros de los encabezamientos de Procesamiento de Datos, Circuitos, Semiconductores y Robots.

La Gráfica 3 nos permite observar las anotaciones anteriores especialmente en cuanto a la nivelación, por lo bajo, de las colecciones en las bibliotecas participantes, frente a la Biblioteca del Tecnológico de Monterrey en esta área de electrónica. 
Cuadro 3. Grupo de Electrónica - Existencias por Bibliotecas 2000 -2006

\begin{tabular}{|c|c|c|c|c|c|c|c|c|c|}
\hline \multirow{2}{*}{$\begin{array}{c}\text { Encabezamiento } \\
\text { de materia }\end{array}$} & \multicolumn{2}{|c|}{$\begin{array}{l}\text { B. Luis ángel } \\
\text { arango }\end{array}$} & \multicolumn{3}{|c|}{$\begin{array}{l}\text { B. Tecnológica } \\
\text { De monterrey }\end{array}$} & \multirow{2}{*}{\begin{tabular}{|c|}
$\begin{array}{c}\text { U. } \\
\text { Andes }\end{array}$ \\
Núm.
\end{tabular}} & \multirow{2}{*}{\begin{tabular}{|c|}
$\begin{array}{c}\text { San } \\
\text { Francisco }\end{array}$ \\
Núm. \\
\end{tabular}} & \multirow{2}{*}{$\begin{array}{c}\text { U. } \\
\text { Javeriana } \\
\text { Núm. }\end{array}$} & \multirow{2}{*}{$\begin{array}{l}\text { UIS } \\
\text { Núm }\end{array}$} \\
\hline & \begin{tabular}{|l|} 
Núm. de \\
Registros \\
Existentes
\end{tabular} & $\begin{array}{l}2000- \\
2006\end{array}$ & Español & Inglés & Total & & & & \\
\hline Autómatas & 0 & 0 & 7 & 9 & 16 & 9 & & 7 & \\
\hline Automatización & 453 & 43 & 54 & 63 & 117 & & & 10 & 4 \\
\hline Circuitos & 889 & 86 & 98 & 336 & 434 & & 4 & 20 & 34 \\
\hline Diseño lógico & 46 & 7 & 5 & 21 & 26 & & 0 & 4 & 5 \\
\hline Hardware & 48 & 2 & 5 & 2 & 7 & 6 & & 3 & \\
\hline $\begin{array}{l}\text { Lógica simbólica } \\
\text { y matemática }\end{array}$ & 267 & 40 & 11 & 13 & 24 & & 0 & 2 & \\
\hline Microprocesadores & 78 & 5 & 8 & 30 & 38 & & 0 & 1 & 5 \\
\hline $\begin{array}{l}\text { Procesamiento } \\
\text { de datos }\end{array}$ & 45 & 2 & 182 & 736 & 918 & & 6 & 25 & 41 \\
\hline Robótica & 99 & 12 & 6 & 85 & 91 & 3 & & 12 & 10 \\
\hline Robots & 153 & 20 & 36 & 155 & 191 & & 87 & 12 & 10 \\
\hline Semiconductores & 178 & 12 & 12 & 107 & 119 & 2 & 3 & 7 & 5 \\
\hline $\begin{array}{l}\text { Sistemas de } \\
\text { potencia }\end{array}$ & 24 & 3 & 0 & 0 & 0 & 4 & & 1 & \\
\hline $\begin{array}{l}\text { Sistemas } \\
\text { dinámicos }\end{array}$ & 40 & 7 & 2 & 15 & 17 & 1 & & 5 & 2 \\
\hline Totales & 2.320 & 239 & 426 & 1.572 & 1.998 & 25 & 100 & 109 & 116 \\
\hline
\end{tabular}

Fuente: elaboración propia

Gráfica 3. Grupo de electrónica 2000 - 2006

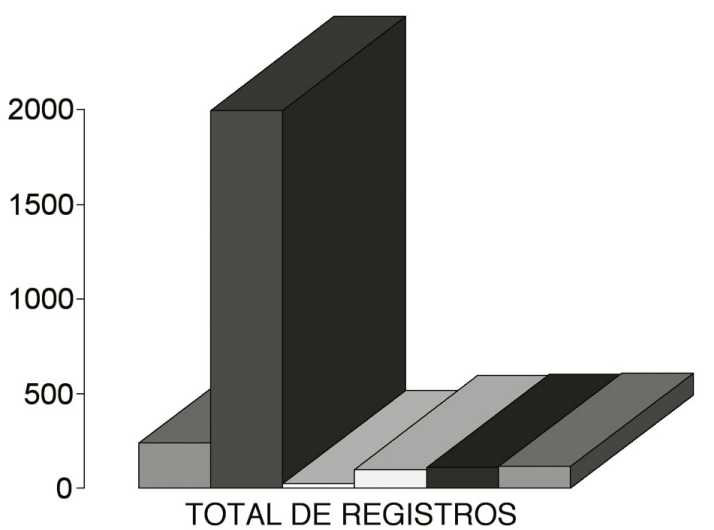

$\square$ B. Luis Angel Arango

B. P. Monterrey

$\square$ B. U. Andes

$\square$ B. P. San Francisco

B. U. Javeriana

a. UIS

Fuente: elaboración propia 


\section{2) Grupo de Redes y Telecomunicaciones - Existencias por Bibliotecas $2000-2006$}

En el Cuadro 4 del grupo de Redes y Telecomunicaciones, podemos observar las colecciones que tienen en el área cada una de las bibliotecas participantes. El mismo cuadro facilita el análisis de las existencias de registros por cada uno de los encabezamientos de materia.

En este grupo es significativa la coincidencia entre los encabezamientos de materia usados por la BLA y las bibliotecas, los del Tecnológico de Monterrey, U. Javeriana y la UIS.

En este grupo de Redes y Telecomunicaciones no se percibe mucha diferencia entre las existencias de libros por encabezamiento de materia entre la Biblioteca del Tecnológico de Monterrey y la BLA; por el contrario, se advierte un equilibrio proporcional en el crecimiento de las colecciones. Igual sucede en cuanto al total de los libros existentes después del 2000, donde se obtienen cifras muy equivalentes, si exceptuamos, Redes de Computadores, donde se manifiesta una clara superioridad de la BLA.

El $73.3 \%$ de los libros que posee la BLA en esta área son anteriores al año 2000. El porcentaje, como en el caso del grupo anterior, es muy alto.

Cuadro 4. Grupo de Redes y Telecomunicaciones - Existencias por Bibliotecas 2000 -2006

\begin{tabular}{|c|c|c|c|c|c|c|c|c|c|}
\hline \multirow{2}{*}{$\begin{array}{l}\text { Encabezamiento } \\
\text { de Materia }\end{array}$} & \multicolumn{2}{|c|}{$\begin{array}{l}\text { B. Luis Ángel } \\
\text { Arango }\end{array}$} & \multicolumn{3}{|c|}{$\begin{array}{l}\text { B. Tecnológica } \\
\text { de Monterrey }\end{array}$} & \multirow{2}{*}{\begin{tabular}{|c|}
$\begin{array}{c}\text { U. } \\
\text { Andes }\end{array}$ \\
Núm. \\
\end{tabular}} & \multirow{2}{*}{\begin{tabular}{c|}
$\begin{array}{c}\text { San } \\
\text { Francisco }\end{array}$ \\
Núm.
\end{tabular}} & \multirow{2}{*}{\begin{tabular}{c|} 
U. \\
Javeriana
\end{tabular}} & \multirow{2}{*}{$\begin{array}{r}\text { UIS } \\
\text { Núm. }\end{array}$} \\
\hline & \begin{tabular}{l|} 
Núm. de \\
Registros \\
Existente \\
\end{tabular} & $\begin{array}{l}2000- \\
2006\end{array}$ & Español & Inglés & Total & & & & \\
\hline $\begin{array}{l}\text { Admininistración } \\
\text { de redes }\end{array}$ & 54 & 33 & 0 & 4 & 4 & 7 & 135 & 6 & 4 \\
\hline $\begin{array}{l}\text { Arquitectura de } \\
\text { redes }\end{array}$ & 28 & 17 & 2 & 11 & 13 & 7 & & 3 & 1 \\
\hline $\begin{array}{l}\text { Comunicaciones } \\
\text { digitales }\end{array}$ & 83 & 15 & 9 & 47 & 56 & & 14 & 6 & 2 \\
\hline Microondas & 61 & 9 & 1 & 5 & 6 & & & 0 & 2 \\
\hline Multimedia & 212 & 81 & 32 & 151 & 183 & 10 & 17 & 16 & 4 \\
\hline $\begin{array}{l}\text { Redes de área } \\
\text { amplia }\end{array}$ & 10 & 7 & 3 & 3 & 6 & & 3 & 1 & \\
\hline $\begin{array}{l}\text { Redes de área } \\
\text { local }\end{array}$ & 141 & 22 & 17 & 36 & 53 & & 6 & 8 & 12 \\
\hline $\begin{array}{l}\text { Redes de } \\
\text { computadores }\end{array}$ & 685 & 179 & 11 & 144 & 155 & & 38 & 56 & 36 \\
\hline $\begin{array}{l}\text { Redes de } \\
\text { informacion }\end{array}$ & 282 & 47 & 5 & 2 & 7 & & 3 & 19 & 1 \\
\hline
\end{tabular}




\begin{tabular}{|l|c|c|c|c|c|c|c|c|c|}
\hline $\begin{array}{l}\text { Redes } \\
\text { inalámbricas }\end{array}$ & 14 & 7 & 3 & 1 & 4 & 0 & & 11 & 2 \\
\hline Redes neuronales & 14 & 7 & 9 & 14 & 23 & 14 & 4 & 14 & 5 \\
\hline \multicolumn{1}{|c|}{ Totales } & $\mathbf{1 . 5 8 4}$ & $\mathbf{4 2 4}$ & $\mathbf{9 2}$ & $\mathbf{4 1 8}$ & $\mathbf{5 1 0}$ & $\mathbf{3 8}$ & $\mathbf{2 2 0}$ & $\mathbf{1 4 0}$ & $\mathbf{6 9}$ \\
\hline
\end{tabular}

Fuente: elaboración propia

También podemos ver en la Gráfica 4 los registros totales por biblioteca, y la participación de cada una de ellas en el monto total de libros existentes en el área.

Gráfica 4. Grupo de Redes y Telecomunicaciones 2000 - 2006

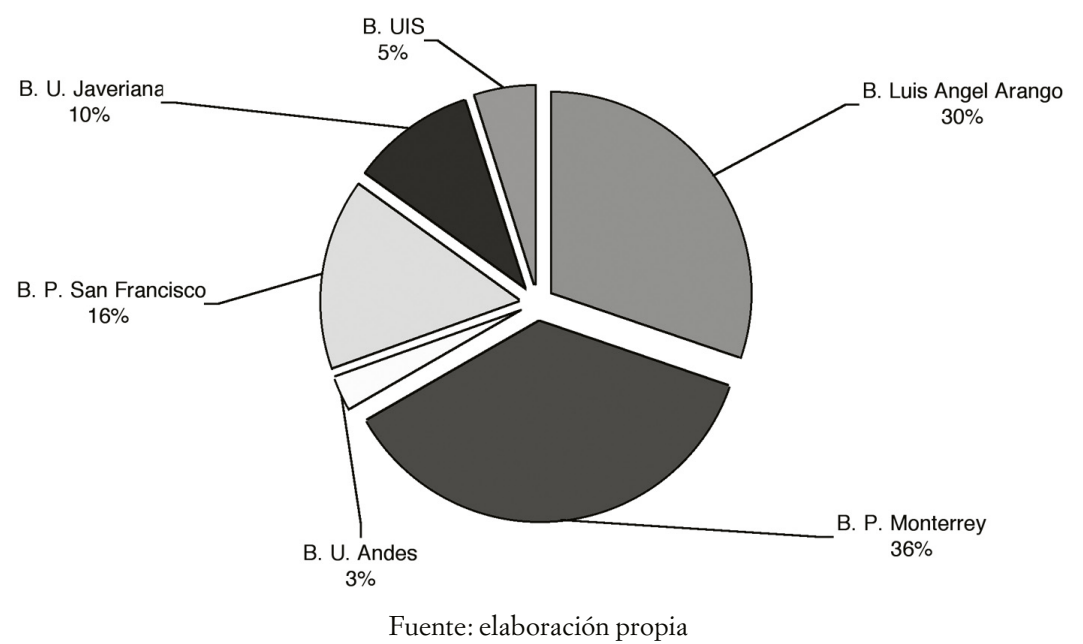

\section{3) Grupo de Bases de Datos - Existencias por Bibliotecas 2000 -2006}

En cuanto a las Bases de Datos, en el Cuadro 5 se aprecia una coincidencia del cien por ciento en cuanto a los encabezamientos de materia, entre la biblioteca BLA y la del Tecnológico de Monterrey. También es apreciable la diferencia en las existencias de las colecciones en el área, dado que la colección que posee la BLA, sólo representa el 13\% del total del grupo, y la de la Biblioteca del Tecnológico de Monterrey representa el 71\% del total de los registros de las seis bibliotecas objeto del estudio. Asimismo observamos que hay claras diferencias en cuanto al número de registros entre la BLA y la biblioteca del Tecnológico de Monterrey, en los encabezamientos de Bases de Datos, Administración de Bases de Datos, Administración de la Información y SQL. 
Cuadro 5. Grupo de Bases de Datos - Existencias por Bibliotecas 2000 -2006

\begin{tabular}{|c|c|c|c|c|c|c|c|c|c|}
\hline \multirow{2}{*}{$\begin{array}{c}\text { Encabezamiento } \\
\text { de materia }\end{array}$} & \multicolumn{2}{|c|}{$\begin{array}{l}\text { B. Luis Ángel } \\
\text { Arango }\end{array}$} & \multicolumn{3}{|c|}{$\begin{array}{l}\text { B. Tecnológica } \\
\text { De monterrey }\end{array}$} & \multirow{2}{*}{$\begin{array}{c}\begin{array}{c}\text { U. } \\
\text { Andes }\end{array} \\
\text { Núm. }\end{array}$} & \multirow{2}{*}{$\begin{array}{c}\begin{array}{c}\text { San } \\
\text { Francisco }\end{array} \\
\text { Núm. }\end{array}$} & \multirow{2}{*}{$\begin{array}{c}\text { U. } \\
\text { Javeriana } \\
\text { Núm. }\end{array}$} & \multirow{2}{*}{\begin{tabular}{|l} 
UIS \\
Núm.
\end{tabular}} \\
\hline & $\begin{array}{l}\text { Núm. de } \\
\text { Registros } \\
\text { Existente }\end{array}$ & $\begin{array}{l}2000- \\
2006\end{array}$ & Español & Inglés & Total & & & & \\
\hline $\begin{array}{l}\text { Admininistración } \\
\text { de bases de } \\
\text { datos }\end{array}$ & 227 & 57 & 67 & 195 & 262 & 22 & 32 & 11 & 9 \\
\hline $\begin{array}{l}\text { Admininistración } \\
\text { de la información }\end{array}$ & 13 & 4 & 38 & 446 & 484 & 2 & 0 & 46 & 9 \\
\hline $\begin{array}{l}\text { Almacenamiento } \\
\text { de información }\end{array}$ & 20 & 1 & 0 & 1 & 1 & 9 & 3 & 18 & 0 \\
\hline Bases de datos & 338 & 64 & 77 & 207 & 284 & & 7 & 16 & 21 \\
\hline $\begin{array}{l}\text { Bases de datos } \\
\text { relacionales }\end{array}$ & 73 & 30 & 23 & 73 & 96 & 2 & & 3 & 15 \\
\hline Bases distribuidas & 13 & 2 & 2 & 5 & 7 & & & 1 & 0 \\
\hline $\begin{array}{l}\text { Diseño de bases } \\
\text { de datos }\end{array}$ & 91 & 33 & 3 & 35 & 38 & 10 & 12 & 10 & 2 \\
\hline Minería de datos & 4 & 4 & 12 & 32 & 44 & 9 & 6 & 4 & 0 \\
\hline $\begin{array}{l}\text { Protección de } \\
\text { datos }\end{array}$ & 129 & 49 & 12 & 38 & 50 & 22 & 11 & 9 & 2 \\
\hline Sql & 75 & 38 & 42 & 207 & 249 & & 26 & 0 & 15 \\
\hline Totales & 983 & 282 & 276 & 1.239 & 1.515 & 76 & 97 & 118 & 73 \\
\hline
\end{tabular}

La Gráfica 5 resume los datos del cuadro y se hacen más evidente para el lector las diferencias entre las colecciones de las bibliotecas participantes.

Gráfica 5. Grupo de Bases de Datos 2000 - 2006

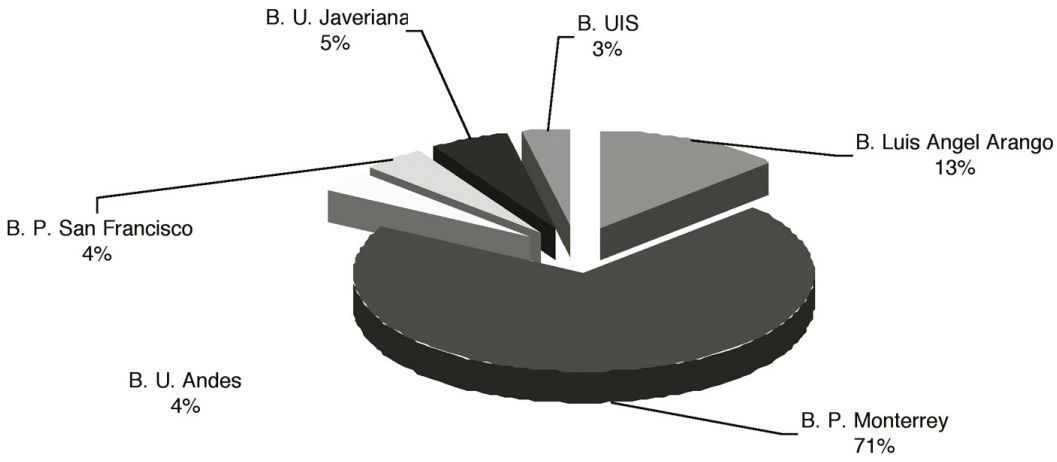

Fuente: elaboración propia 
Así, la Base de Datos del Tecnológico de Monterrey aparece con un 7\%, seguida a distancia por la BLA y del resto de las instituciones.

\section{4) Grupo de Sistemas Informáticos - Existencias por Bibliotecas 2000 -2006}

Con respecto al Grupo de Sistemas Informáticos (Cuadro 6), podemos observar que se conserva la tendencia, hacia un mayor crecimiento de la colección del año 2000 en adelante en la Biblioteca del Tecnológico de Monterrey, frente a la BLA. En el mismo sentido, en esta área, la Biblioteca de la Universidad Javeriana tiene un crecimiento comparable con la colección que posee la BLA.

En el grupo hay tres encabezamientos de la BLA, que la Biblioteca del Tecnológico de Monterrey no utiliza: Computadores - Historia, Ingenieros de Sistemas, y Traductores, al menos este último, con el sentido que tiene en sistemas.

La colección de la BLA antes del año 2000 representa el 85\% del total de sus existencias, el cual es alto si tenemos en cuanta los encabezamientos de materia que este grupo contiene (Gráfica 6). (En la Gráfica 6 se visualiza con detalle la situación de esta área).

Cuadro 6. Grupo de Sistemas Informáticos - Existencias por Bibliotecas 2000 -2006

\begin{tabular}{|c|c|c|c|c|c|c|c|c|c|}
\hline \multirow{2}{*}{$\begin{array}{c}\text { Encabezamiento } \\
\text { de Materia }\end{array}$} & \multicolumn{2}{|c|}{$\begin{array}{l}\text { B. Luis Ángel } \\
\text { Arango }\end{array}$} & \multicolumn{3}{|c|}{$\begin{array}{l}\text { B. Tecnológica } \\
\text { de Monterrey }\end{array}$} & \multirow[t]{2}{*}{$\begin{array}{c}\text { U. } \\
\text { Andes }\end{array}$} & \multirow[t]{2}{*}{$\begin{array}{c}\text { San } \\
\text { Francisco }\end{array}$} & \multirow[t]{2}{*}{\begin{tabular}{c|}
$\mathrm{U}$. \\
Javeriana
\end{tabular}} & \multirow[t]{2}{*}{ UIS } \\
\hline & $\begin{array}{l}\text { Núm. de } \\
\text { Registros } \\
\text { Existente }\end{array}$ & $\begin{array}{l}2000- \\
2006\end{array}$ & Español & Inglés & Total & & & & \\
\hline Compiladores & 15 & 1 & 2 & 17 & 19 & 0 & 0 & 1 & 0 \\
\hline Computadores & 1524 & 161 & 3 & 12 & 15 & & 21 & 155 & 120 \\
\hline $\begin{array}{l}\text { Computadores - } \\
\text { Historia }\end{array}$ & 76 & 14 & & & & 1 & & 4 & 0 \\
\hline $\begin{array}{l}\text { Delitos por } \\
\text { computadores }\end{array}$ & 50 & 17 & 4 & 7 & 11 & 9 & 6 & 2 & \\
\hline $\begin{array}{l}\text { Informática - } \\
\text { aspectos sociales }\end{array}$ & 25 & 3 & 27 & 6 & 33 & 21 & 1 & 1 & \\
\hline $\begin{array}{l}\text { Ingeniería de } \\
\text { sistemas }\end{array}$ & 112 & 11 & 27 & 54 & 81 & & 2 & 2 & 0 \\
\hline $\begin{array}{l}\text { Ingeniería de } \\
\text { software }\end{array}$ & 108 & 12 & 21 & 139 & 160 & 0 & 1 & 18 & 6 \\
\hline $\begin{array}{l}\text { Ingenieros de } \\
\text { sistemas }\end{array}$ & 13 & 1 & & & & & 1 & & \\
\hline $\begin{array}{l}\text { Lenguajes de } \\
\text { programacion }\end{array}$ & 242 & 56 & 14 & 62 & 76 & 23 & 123 & 99 & 3 \\
\hline $\begin{array}{l}\text { Lenguajes } \\
\text { formales }\end{array}$ & 3 & 0 & 1 & 7 & 8 & & & & 2 \\
\hline
\end{tabular}




\begin{tabular}{|l|c|c|c|c|c|c|c|c|c|}
\hline $\begin{array}{l}\text { Lingüística } \\
\text { computacional }\end{array}$ & 8 & 2 & 1 & 9 & 10 & 0 & 0 & 1 & \\
\hline $\begin{array}{l}\text { Programacion } \\
\text { orientada a } \\
\text { objetos }\end{array}$ & 181 & 51 & 50 & 200 & 250 & 6 & 27 & 27 & 9 \\
\hline Teoria de sistemas & 31 & 18 & 10 & 16 & 26 & 0 & 3 & 4 & 2 \\
\hline Traductores & 26 & 8 & & & & & & & 0 \\
\hline \multicolumn{1}{|c|}{ Totales } & $\mathbf{2 . 4 1 4}$ & $\mathbf{3 5 5}$ & $\mathbf{1 6 0}$ & $\mathbf{5 2 9}$ & $\mathbf{6 8 9}$ & $\mathbf{6 0}$ & $\mathbf{1 8 5}$ & $\mathbf{3 1 4}$ & $\mathbf{1 4 2}$ \\
\hline
\end{tabular}

Gráfica 6. Grupo de Sistemas Informáticos 2000 - 2006

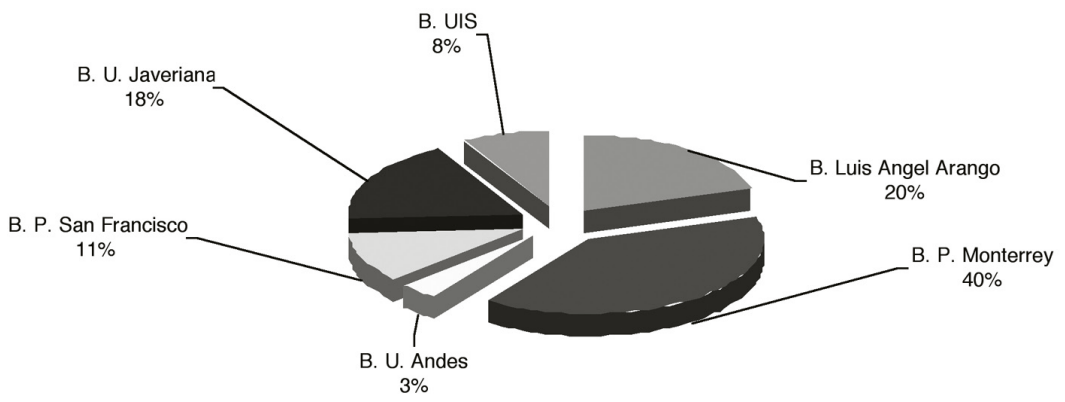

Fuente: elaboración propia

\section{5) Grupo de Aplicaciones Informáticas - Existencias por Bibliotecas} $2000-2006$

En el Cuadro 7 del grupo de Aplicaciones Informáticas se puede advertir que es donde hay más desequilibrio entre las dos bibliotecas del exterior con la BLA, el cual se acentúa más todavía para las bibliotecas de las universidades colombianas.

Cuadro 7. Grupo de Aplicaciones Informáticas - Existencias por Bibliotecas 2000 -2006

\begin{tabular}{|c|c|c|c|c|c|c|c|c|c|}
\hline \multirow{2}{*}{$\begin{array}{c}\text { Encabezamiento } \\
\text { de Materia }\end{array}$} & \multicolumn{2}{|c|}{$\begin{array}{l}\text { B. Luis Ángel } \\
\text { Arango }\end{array}$} & \multicolumn{3}{|c|}{$\begin{array}{l}\text { B. Tecnológica } \\
\text { de Monterrey }\end{array}$} & \multirow{2}{*}{$\begin{array}{c}\begin{array}{c}\text { U. } \\
\text { Andes }\end{array} \\
\text { Núm. }\end{array}$} & \multirow{2}{*}{$\begin{array}{c}\text { San } \\
\text { Francisco } \\
\text { Núm. }\end{array}$} & \multirow{2}{*}{$\begin{array}{c}\text { U. } \\
\text { Javeriana } \\
\text { Núm. }\end{array}$} & \multirow{2}{*}{$\begin{array}{l}\text { UIS } \\
\text { Núm. }\end{array}$} \\
\hline & $\begin{array}{l}\text { Núm. de } \\
\text { Registros } \\
\text { Existente }\end{array}$ & $\begin{array}{l}2000- \\
2006\end{array}$ & Español & Inglés & Total & & & & \\
\hline $\begin{array}{l}\text { Análisis de } \\
\text { sistemas }\end{array}$ & 288 & 35 & 35 & 120 & 155 & & 1 & 21 & 9 \\
\hline $\begin{array}{l}\text { Gráficos por } \\
\text { computador }\end{array}$ & 476 & 64 & 41 & 136 & 177 & 24 & 218 & 19 & 7 \\
\hline Metadatos & 10 & 2 & 1 & 7 & 8 & & 1 & 1 & \\
\hline Microsoft & 614 & 251 & 511 & 1000 & 1511 & & 596 & 23 & 4 \\
\hline
\end{tabular}




\begin{tabular}{|l|c|c|c|c|c|c|c|c|c|}
\hline Realidad virtual & 121 & 27 & 21 & 30 & 51 & 5 & 7 & 16 & 0 \\
\hline $\begin{array}{l}\text { Simulación por } \\
\text { computadora }\end{array}$ & 81 & 17 & 15 & 173 & 188 & 7 & 24 & 12 & 25 \\
\hline $\begin{array}{l}\text { Sistemas de } \\
\text { archivo }\end{array}$ & 2 & 2 & 0 & 1 & 1 & 13 & & & \\
\hline \multicolumn{1}{|c|}{ Totales } & $\mathbf{1 . 5 9 2}$ & $\mathbf{3 9 8}$ & $\mathbf{6 2 4}$ & $\mathbf{1 . 4 6 7}$ & $\mathbf{2 . 0 9 1}$ & $\mathbf{4 9}$ & $\mathbf{8 4 7}$ & $\mathbf{9 2}$ & $\mathbf{4 5}$ \\
\hline
\end{tabular}

El peso del desequilibrio fundamentalmente esta en el encabezamiento de materia Microsoft, que en la Biblioteca del Tecnológico de Monterrey, a partir del año 2000, tiene un total de 1511 registros y en la Biblioteca Pública de San Francisco es de 596; mientras que la BLA sólo posee 251 registros, lo que equivaldría a tener sólo 1/6 parte de lo que tiene la Biblioteca del Tecnológico de Monterrey. Las bibliotecas universitarias colombianas tienen muy pocos registros y la única biblioteca que no usa este encabezamiento es la Biblioteca de la Universidad de los Andes.

También existe una diferencia apreciable entre la Biblioteca del Tecnológico de Monterrey y la BLA en tres encabezamientos que requieren permanente actualización: Gráficos por Computadora, Análisis por Computadora y Simulación por Computadora.

Gráfica 7. Grupo de Aplicaciones Informáticas 2000 - 2006

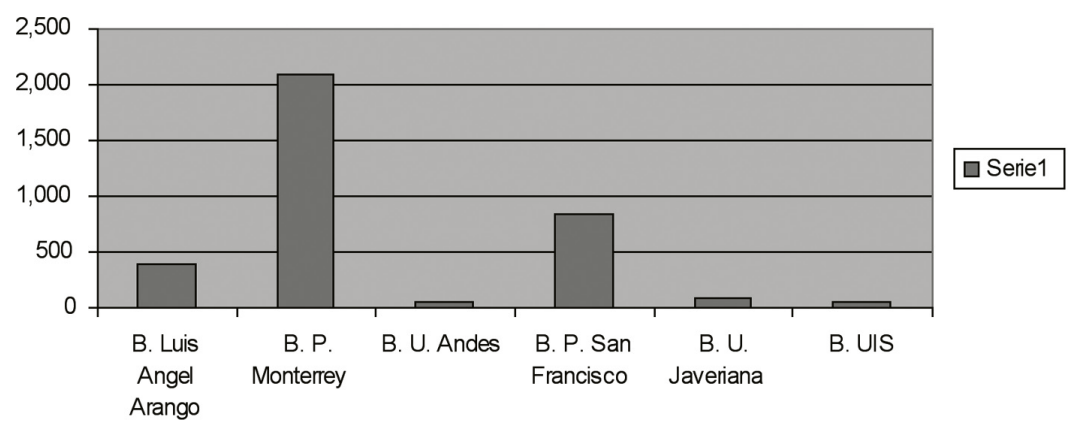

Fuente: elaboración propia

La Gráfica 7 nos muestra la aportación de cada biblioteca en esta área, y evidencia las diferencias en las colecciones de las dos bibliotecas del exterior frente a las del país.

\section{6) Grupo de Internet - Existencias por Bibliotecas 2000-2006}

En el Grupo de Internet (Cuadro 8), se observa que hay dos encabezamientos que sostienen todo el peso de la contribución: Internet, la Biblioteca del 
Tecnológico de Monterrey posee un total de 1657 registros, mientras que la BLA sólo tiene 417, o sea, una cuarta parte de los registros que tiene la de Monterrey, y está igualmente por debajo de los registros que posee la Biblioteca Pública de San Francisco.

Cuadro 8. Grupo de Internet - Existencias por Bibliotecas 2000 -2006

\begin{tabular}{|c|c|c|c|c|c|c|c|c|c|}
\hline \multirow{2}{*}{$\begin{array}{l}\text { Encabezamiento } \\
\text { de materia }\end{array}$} & \multicolumn{2}{|c|}{$\begin{array}{l}\text { B. Luis Ángel } \\
\text { Arango }\end{array}$} & \multicolumn{3}{|c|}{$\begin{array}{l}\text { B. Tecnológica } \\
\text { de Monterrey }\end{array}$} & \multirow{2}{*}{$\begin{array}{c}\begin{array}{c}\text { U. } \\
\text { Andes }\end{array} \\
\text { Núm. }\end{array}$} & \multirow{2}{*}{\begin{tabular}{|c|}
$\begin{array}{c}\text { San } \\
\text { Francisco }\end{array}$ \\
Núm.
\end{tabular}} & \multirow{2}{*}{\begin{tabular}{|c|}
$\begin{array}{c}\text { U. } \\
\text { Javeriana }\end{array}$ \\
Núm.
\end{tabular}} & \multirow{2}{*}{\begin{tabular}{|l} 
UIS \\
Núm.
\end{tabular}} \\
\hline & $\begin{array}{l}\text { Núm. de } \\
\text { Registros } \\
\text { Existente }\end{array}$ & $\begin{array}{l}2000- \\
2006\end{array}$ & Español & Inglés & Total & & & & \\
\hline Internet & 1280 & 417 & 397 & 1260 & 1657 & 7 & 584 & 110 & 18 \\
\hline Navegadores & 40 & 13 & 0 & 0 & 0 & & & 1 & \\
\hline Páginas web & 79 & 53 & 29 & 11 & 40 & & & 3 & \\
\hline $\begin{array}{l}\text { Sistemas } \\
\text { hipertexto }\end{array}$ & 50 & 13 & 7 & 1 & 8 & & & 11 & 0 \\
\hline $\begin{array}{l}\text { World Wide Web } \\
\text { red servicios }\end{array}$ & 6 & 4 & & & 0 & & & & \\
\hline $\begin{array}{l}\text { World Wide } \\
\text { Web red de } \\
\text { computadoras }\end{array}$ & 4 & 2 & 36 & 100 & 136 & 12 & 63 & 42 & 3 \\
\hline Totales & 1.459 & 502 & 469 & 1.372 & 1.841 & 19 & 647 & 167 & 21 \\
\hline
\end{tabular}

Con respecto a este grupo la BLA usa dos encabezamientos de materia para la World Wide Web con subdivisiones, contrariamente a las demás instituciones que sólo usan una subdivisión. El encabezamiento de materia Navegadores, usado en la BLA, fue utilizado también en la Biblioteca del Tecnológico de Monterrey hasta antes del año 2000.

La Gráfica 8 nos permite ver las contribuciones que hizo cada una de las bibliotecas; llama la atención el bajo número de aportaciones que hicieron las bibliotecas de las universidades de los Andes y la UIS. 
Gráfica 8. Grupo de Internet 2000 - 2006

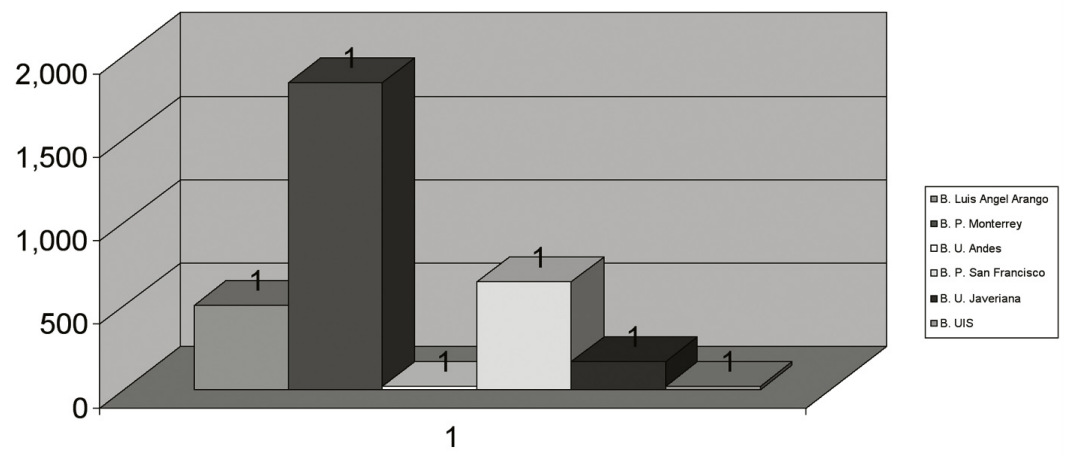

Fuente: elaboración propia

\section{7) Análisis de los registros contenidos en los encabezamientos que no se usaron en el estudio comparativo 9}

Como ya se mencionó, la recolección de datos en todas las bibliotecas del estudio, se hizo con base en los 62 encabezamientos registrados, pero en la búsqueda realizada en las bases de datos de las diferentes bibliotecas participantes se encontraron 20 encabezamientos de materia que no forman parte de los 62 ya enunciados. Estos encabezamientos se encontraron en las bibliotecas de las universidades Javeriana, los Andes, la UIS y en la Biblioteca del Tecnológico de Monterrey.

Cuadro 9. Colecciones en los encabezamientos de las bibiotecas no usados en la biblioteca Luis Ángel Arango

\begin{tabular}{|l|c|c|c|c|}
\hline \multicolumn{1}{|c|}{$\begin{array}{c}\text { Encabezamiento } \\
\text { de Materia }\end{array}$} & $\begin{array}{c}\text { B. Univ. } \\
\text { Javeriana }\end{array}$ & $\begin{array}{c}\text { B. Tec. } \\
\text { Monterrey }\end{array}$ & B. L. A. A. & $\begin{array}{c}\text { B Universidad } \\
\text { de Los Andes }\end{array}$ \\
\hline $\begin{array}{l}\text { Arquitectura de } \\
\text { computadoras }\end{array}$ & 13 & 3 & 3 \\
\hline Computación movil & & 12 & 2 & 1 \\
\hline $\begin{array}{l}\text { Diseño de interfaces } \\
\text { gráficas }\end{array}$ & 25 & 121 & 1 & \\
\hline Diseño de sistemas & & 173 & & \\
\hline Procesamiento de señales & 10 & & & \\
\hline Estructura de datos & 41 & & & \\
\hline Inteligencia artificial & 3 & & & \\
\hline Lasers & 7 & & & \\
\hline Microcomputadoras & & & & \\
\hline
\end{tabular}

9 La afirmación que estos encabezamientos no son usados en la Biblioteca Luís Ángel Arango se hace con base en la lista oficial remitida de encabezamientos de materia usados por parte de la biblioteca. 


\begin{tabular}{|l|c|l|c|c|}
\hline Sistemas expertos & 4 & & & \\
\hline Teoría control & 4 & & & 0 \\
\hline Ciencia computacional & & & & 47 \\
\hline $\begin{array}{l}\text { Documentación de } \\
\text { programas }\end{array}$ & & & & 10 \\
\hline Hipertexto e hipermedia & & & & 4 \\
\hline $\begin{array}{l}\text { Programación de interfaces } \\
\text { gráficas }\end{array}$ & & & & 15 \\
\hline Programación concurrente & & & & 31 \\
\hline $\begin{array}{l}\text { Propiedad intelectual de } \\
\text { software }\end{array}$ & & & & 3 \\
\hline Redes alámbricas & & & & 7 \\
\hline Redes y comunicaciones & & & & 19 \\
\hline Sistemas inteligentes & & & & 22 \\
\hline Seguridad de datos & & & & 0 \\
\hline $\begin{array}{l}\text { Sistemas manejadores de } \\
\text { bases de datos }\end{array}$ & & & & 10 \\
\hline Teletráfico & & & & $\mathbf{1 7 2}$ \\
\hline $\begin{array}{l}\text { Tratamiento digital de } \\
\text { imágenes }\end{array}$ & & & & 0 \\
\hline \multicolumn{1}{|l|}{ Total de registros } & $\mathbf{9 4}$ & & & \\
\hline
\end{tabular}

Fuente: elaboración propia

Estos encabezamientos y sus respectivas colecciones (del año 2000 en adelante) fueron separados del estudio central con el objeto de crear un archivo independiente. (Cuadro 9) lo que le permitiría a la BLA evaluar tanto los encabezamientos de materia como las colecciones existentes en cada uno de los nuevos temas.

En el Cuadro 9 se puede observar que la Biblioteca de la Universidad Javeriana tiene 94 registros del año 2000 en adelante en siete encabezamientos de materia; la Biblioteca de la Universidad de los Andes tiene 172 registros en quince encabezamientos de materia, (pero señalemos que en tres de ellos no hay ningún registro a partir del año 2000 en adelante) la Biblioteca de la UIS tiene seis registros en tres encabezamientos de materia y la Biblioteca del Tecnológico de Monterrey posee 344 registros en cinco encabezamientos de materia.

Cabe señalar, como se puede observar en el cuadro mencionado, que en estos encabezamientos hallados, sólo en cuatro de ellos hay coincidencia entre las bibliotecas estudiadas: Diseño de Sistemas, en el cual coinciden las Bibliotecas de la U. Javeriana, la UIS y la del Tecnológico de Monterrey; Diseño de Interfaces Gráficas, en el que coinciden las Bibliotecas de la UIS y el Tecnológico de Monterrey; Computación Móvil, en el que concuerdan las 
dos anteriores, y la Biblioteca de la Universidad de los Andes; Arquitectura de Computadores, con el que concuerdan las bibliotecas del Tecnológico de Monterrey y la de la Universidad de los Andes.

Si tenemos en cuenta el bajo número de registros del año 2000 en adelante en algunas de las bibliotecas participantes en el estudio, cabría esperar que en estos 20 encabezamientos surgieran cifras que mostraran una mayor colección en las bibliotecas participantes. Pero, como se puede observar en el Cuadro 9, en estos 20 encabezamientos sólo hay 616 registros de este periodo, y de ellos 344, el 55\% del total, pertenecen a la Biblioteca del Tecnológico de Monterrey, por tanto, la contribución de las tres bibliotecas restantes no es muy significativa en el total de las colecciones en las áreas objeto de estudio.

\section{Recomendación De AdQUisiciones}

Tal como se describe en la metodología del estudio, después de un proceso de análisis comparativo entre las bases de datos de las bibliotecas objeto de análisis, con bibliografías y catálogos, se construyó con los 62 encabezamientos una base de datos en la que se integraron seis grupos con los libros seleccionados para cada encabezamiento de materia.

Nos preguntamos cuál podría ser un número ideal recomendable para estas áreas tomando en cuenta el presupuesto disponible. La BLA estableció que la cifra podía rondar los 600 libros. Esta indicación contribuyó a nuestra toma de decisión y pudimos así ponderar algunos de los criterios que a lo largo de la revisión fuimos adoptando. Evitamos de este modo una bibliografía extensa, que por una parte exigiera una nueva selección y por la otra dejara un remanente de libros seleccionados sin uso; extremo no contemplado en este trabajo

Para cada encabezamiento de materia empleamos criterios diferentes de selección de los libros que incluiríamos, tales como: vigencia e importancia del tema; importancia del libro en relación con los otros libros que sobre el mismo tema ya existen en la biblioteca; tipo de material; libros recientes de congresos, conferencias, etcétera, relación del libro en cuestión con otros libros de mismo autor, y libros que tratan un tema poco desarrollado por otros autores. Asimismo la selección tuvo en cuenta la vigencia y el equilibrio por temas en el conjunto de la selección, tal como puede verse en el Cuadro 10 y en la Gráfica 10. 
Cuadro 10. Libros recomendados para adquirir (por grupos del área de sistemas, electrónica y telecomunicaciones)

\begin{tabular}{|l|c|}
\hline \multicolumn{1}{|c|}{ Encabezamiento de materia } & Número de libros \\
\hline Electrónica & 138 \\
\hline Redes y telecomunicaciones & 67 \\
\hline Sistemas informáticos & 124 \\
\hline Bases de datos & 76 \\
\hline Aplicaciones informáticas & 93 \\
\hline Internet $\quad 64$ \\
\hline \multicolumn{2}{|c|}{ Total del área } \\
\hline
\end{tabular}

Fuente: elaboración propia

Grafica 10. Áreas/Libros

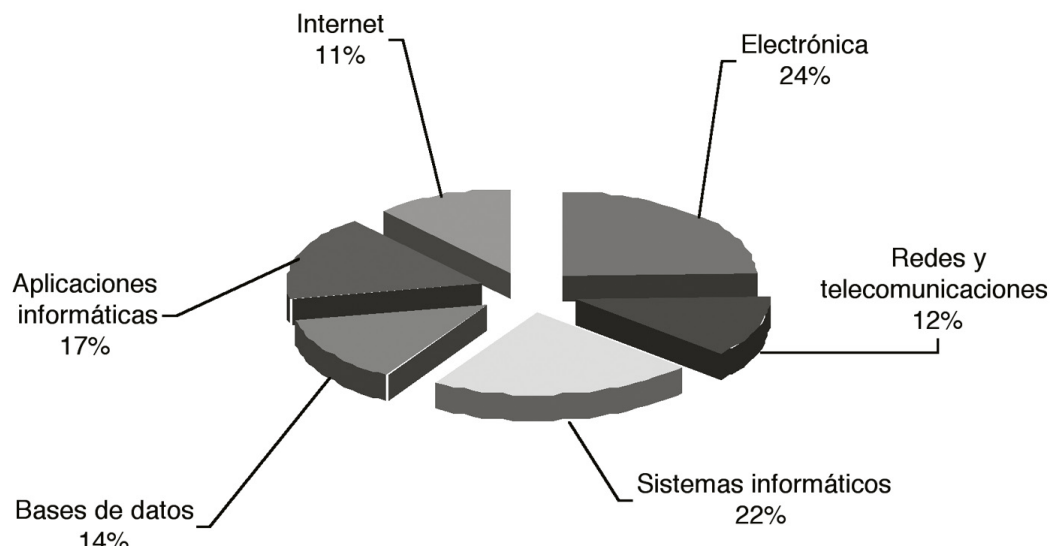

Fuente: elaboración propia

El Cuadro 10 y la Gráfica 10, presentan el resumen en las seis áreas de los libros recomendados a la BLA para su adquisición. ${ }^{10}$ En la gráfica se puede apreciar el equilibrio que se quiso tener al recomendar libros que contienen conocimiento actualizado.

La colección recomendada, 562 libros, representa, en número de libros, el $18.55 \%$ de la colección más completa del estudio, esto es, la del Tecnológico de Monterrey, que a la fecha de la investigación, en los 62 encabezamientos

10 Si la BLA quisiera ampliar en algunas áreas el rango de sus adquisiciones, podría recurrir a las bases de datos de la Biblioteca del Tecnológico de Monterrey creada como resultado del estudio o la de cualquier otra de las bibliotecas, consultando por encabezamiento de materia en los archivos que a ella le fueron entregados. Igualmente se entregó, para cada uno de los encabezamientos de materia, un archivo en Excel, en donde se describe cada libro recomendado con sus datos de autor, título y pie de imprenta, de acuerdo con las normas de la descripción catalográfica. 
tenía 9,088 registros, que equivaldrían, tomando como base 3 encabezamientos por libro, 3.029 libros. Si comparamos estos datos con los 2.088 registros posteriores al 2000 que tiene la BLA, estos equivaldrían, según la base establecida, a 762 libros. Si sumamos los libros recomendados a estos 762 en las áreas objeto de estudio, nos quedaría un total de 1.324, lo que correspondería a un $43.71 \%$ de la colección actual que posee la Biblioteca del Tecnológico de Monterrey, cuando en el momento es sólo del 25.15\%.

\section{Conclusiones}

\section{En cuanto a la metodología del estudio y los resultados}

Las dos herramientas metodológicas utilizadas por los autores en el presente estudio se inspiran ${ }^{11}$ entre otras fuentes consultadas, en dos metodologías, la de "Evaluación de Colecciones en sí mismas" y la de "Gestión de Calidad de Procesos, utilizando técnicas de Benchmarking”.

La evaluación de las colecciones de la BLA, confrontada con las colecciones de las bibliotecas seleccionadas por su prestigio y reconocimiento, se proponía obtener datos que permitieran valorar la colección. La consulta mostró que sólo la Biblioteca del Tecnológico de Monterrey tenía mejores indicadores que la BLA en sus colecciones y en la actualización de las mismas. También se constató que el desarrollo de ésta era equiparable, en estas áreas, a una de las más prestigiosas bibliotecas públicas de los Estados Unidos, la Biblioteca Pública de San Francisco.

Se evidenció que las bibliotecas del país seleccionadas para el estudio estaban en un nivel inferior, en cuanto a sus colecciones, a las de la BLA. El hecho de haber comparado la BLA con otras bibliotecas pares y líderes presentó otras ventajas adicionales. De hecho se encontraron al menos dos bibliotecas pares con las que puede confrontar sistemáticamente sus colecciones y procesos a lo largo del tiempo, para analizar avances o retrocesos, compatibilidades e incompatibilidades. Igual acción podrían seguir las bibliotecas colombianas incluidas en el estudio.

Confrontar la colección de libros de la BLA con la colección más fuerte, la del Tecnológico de Monterrey, y cotejar sus encabezamientos con criterios académicos revisando cada uno de los resultados que el metabuscador iba arrojando, en cuanto a la calidad de cada uno de los libros a incluir, produjo

11 Se mencionan como herramientas inspiradoras dado que ellas no fueron utilizadas rigurosamente en sus técnicas, procedimientos y sistemas formalmente reconocidos. 
una lista de libros preliminar que la Biblioteca debería adquirir, si quiere alcanzar un ajuste en la calidad y cantidad de sus colecciones.

La lista resultante se comparó con listas de libros, bibliografías, catálogos de editoriales y fuentes secundarias reconocidas en las áreas del estudio. El objetivo era conformar, a partir de esta confrontación, la lista definitiva que debía recomendarse, lo cual obligó a los investigadores a tomar decisiones con base a parámetros de calidad, actualidad del conocimiento, conveniencia frente a otras áreas, publicaciones de difícil consecución, etcétera.

Se han creado archivos individuales de libros del 2000 en adelante, por encabezamientos de materia. Si la BLA, o cualquiera de las bibliotecas colombianas, lo considerara conveniente podría tomar más registros, pues de estos archivos sólo se obtuvo el equivalente al $18.55 \%$ de la colección más completa del estudio, la del Tecnológico de Monterrey, que como ya se dijo a la fecha de la investigación tenía, en los 62 encabezamientos, 9,088 registros, que equivalen, tomado la base de 3 encabezamientos por libro, a 3.029 libros.

Otro valor agregado de la metodología es poder encontrar encabezamientos no usados por la BLA, lo que en el caso del estudio dejó 20 encabezamientos y 616 registros, de los cuales se puede hacer una selección para nuevas adquisiciones.

\section{En cuanto a la conceptualización del estudio}

Como se mencionó en el apartado 1, el estudio partió de la premisa conceptual de que la BLA tenía características especiales al conjugar por una parte, rasgos de una biblioteca pública y a su vez los de una biblioteca académica para diferentes niveles de comunidades educativas, y por la otra parte, tratarse de evaluar una colección de Sistemas, Redes y Telecomunicaciones, áreas del conocimiento que están en constante cambio y poseen una alta tendencia a la obsolescencia.

Esto se corroboró con los datos obtenidos en el estudio: la colección en las áreas de estudio y en los encabezamientos utilizados son muy similares a las de la Biblioteca del Tecnológico de Monterrey, que es una institución académica de primer orden en tecnologías en América Latina, y al mismo tiempo es igualmente similar a la de la Biblioteca Pública de San Francisco.

En este mismo aspecto se ratifica que la colección de la BLA es la más completa en estas áreas frente a las colecciones de las bibliotecas colombianas incluidas en el trabajo. Por tanto, sus colecciones responden adecuadamente a las necesidades de las diferentes comunidades académicas y a las del público en general, teniendo en cuenta que estas tecnologías son de dominio de todo tipo de usuarios. 
Igualmente el estudio demostró que aunque la BLA está muy por encima de los indicadores de las bibliotecas colombianas, hay áreas, como ya se mencionó en el análisis de los datos, en las que requiere actualización de sus colecciones, teniendo en cuenta la alta tasa de obsolescencia que presentan estas áreas académicas.

\section{En cuanto al material a descartar}

De los 2.810 libros, que aproximadamente corresponden a los 8.430 registros, que la Biblioteca posee anteriores al año 2000, parecería recomendable hacer un descarte que tenga en cuenta, para este caso específico, los siguientes criterios:

- Descartar selectivamente los libros anteriores a 1980, con salvedad de los textos teóricos que abordan tópicos sobre el conocimiento de estas ingenierías, en cualquiera de sus manifestaciones.

- Descartar títulos duplicados anteriores a 1990, dejando cuando más uno o dos ejemplares.

- Descartar ediciones sobreseídas. (Sustituidas por otras más completas y actualizadas), con excepción de aquellas obras donde haya una sucesión histórica del conocimiento.

\section{Bibliografía Consultada}

Alonso Arévalo, Julio, Echeverría Cubillas, M.J. y S. Martín Cerro, «La gestión de las bibliotecas universitarias: indicadores para su evaluación», en Indicadores en la universidad: información y decisiones, Madrid, Ministerio de Educación y Cultura, 1999, pp. 479491, < http://eprints.rclis.org/archive/00000492/>

Alonso Arévalo, Julio y Martín Cerro, Sonia (2000), Benchmarking : una herramienta para gestionar la excelencia en las bibliotecas y los servicios de información, en Proceedings Jornadas de Bibliotecas Universitarias de Castilla y León (3as : 2000 : Burgos) 3, Burgos, http://eprints.rclis.org/view/people/Alonso_Ar=valo,_Julio. html

Babini, D.; Fraga, G, Bibliotecas virtuales para las ciencias sociales, Buenos Aires, CLACSO, 2004.

Comisión Europea, 2010 Digital Library, Luxemburgo, Space Innovation and Investisement, 2007. 
Descarte de libros en bibliotecas públicas: orientaciones generales, Caracas: Biblioteca Nacional, Dirección de Bibliotecas Públicas, Unidad de Normalización Técnica, 1997. 12 p. (Serie: Traducciones); Publicación Normativa Núm., BP -007-96. Traducción, adaptación y adiciones de Luisa Villalba, ISBN 980-319-094-6

Ellis, Martin, «Benchmarking public libraries: Comparisons in context», en APLIS, vol. 11, núm. 2, pp. 56-62, Jun. 98.

Ferrar Sapena, A., Guía metodológica para la implantación de una Biblioteca digital universitaria, Gijón, TREA, 2005.

Massísimo i Sánchez de Boado, Àngels, "Evaluación de colecciones en las bibliotecas universitarias (I). Métodos basados en el uso de la colección", en Anales de Documentación, núm. 5, pags. 245-272, 2002.

Massísimo i Sánchez de Boado, Àngels. "Evaluación de colecciones en las bibliotecas universitarias (II). Métodos basados en el uso de la colección", en Anales de Documentación, núm. 7, pags. 171-183, 2004.

Pontificia Universidad Católica De Chile, Sistema De Bibliotecas. Estudio Comparativo entre Bibliotecas Universitarias Chilenas, Latinoamericanas y estadounidenses. Santiago, Chile, 1999. 20 p.

\section{Catálogos de Bibliotecas Consultados}

Biblioteca Digital del Tecnológico de Monterrey, http://biblioteca. itesm.mx/nav/>

Biblioteca Luís Ángel Arango, http://www.lablaa.org/home.htm>

Biblioteca Pública de San Francisco, <http://sfpl.lib.ca.us/spanish/> http://sflib1.sfpl.org/search*spi/X?NOSRCH=ADMINISTRACION+D $\mathrm{E}+\mathrm{BASES}+\mathrm{DE}+\mathrm{DATOS} \& \mathrm{SORT}=\mathrm{D}$

Biblioteca de la Universidad de los Andes, <http://biblioteca.uniandes.edu.co/>

Biblioteca de la Universidad Javeriana, Bogotá, $<$ http://www.javeriana.edu.co/biblos/bibliote.html>

Biblioteca Universidad Industrial de Santander -UIS-, $<$ https://www.uis.edu.co/portal/biblioteca/index.html>

Fuentes Internacionales de libros

CHOICE, <http://www.choice.com.au/defaultView.aspx?id=102314\& catId $=100165>$

ISBN Español, <http://www.booksfactory.com/isbn1.html>

The Publishers> Trade List Annual (PTLA), <http://palimpsest.stanford.edu/byform/mailing-lists/exlibris/1995/05/msg00011.html> 